\title{
Some characteristic properties of analytic functions
}

\author{
R. K. Raina, Poonam Sharma and G. S. Sălăgean
}

\begin{abstract}
In this paper, we consider a class $\mathcal{L}(\lambda, \mu ; \phi)$ of analytic functions $f$ defined in the open unit disk $\mathbb{U}$ satisfying the subordination condition that

$$
q(z) \frac{\mathcal{D}^{\lambda+1} f(z)}{\mathcal{D}^{\lambda} f(z)} \prec \phi(z)\left(\lambda \in \mathbb{N}_{0}, \mu \geq 0 ; z \in \mathbb{U}\right),
$$

where $q(z)=\left(\frac{z}{\mathcal{D}^{\lambda} f(z)}\right)^{\mu-2}, \mathcal{D}^{\lambda}$ is the Sălăgean operator and $\phi(z)$ is a convex function with positive real part in $\mathbb{U}$. We obtain some characteristic properties giving the coefficient inequality, radius and subordination results, and an inclusion result for the above class when the function $\phi(z)$ is a bilinear mapping in the open unit disk. For these functions $f(z)$, sharp bounds for the initial coefficient and for the Fekete-Szegö functional are determined, and also some integral representations are given.
\end{abstract}

\section{Introduction}

Let $\mathcal{A}$ denote a class of functions $f$ analytic in the open unit disk $\mathbb{U}=\{z:|z|<1\}$ with the normalization that $f(0)=0=f^{\prime}(0)-1$, that is the function $f$ has the series expansion

$$
f(z)=z+\sum_{k=1}^{\infty} a_{k+1} z^{k+1}, z \in \mathbb{U} .
$$

Key Words: Analytic functions, subordination, starlike function, Fekete-Szegö functional, Sălăgean operator.

2010 Mathematics Subject Classification: Primary 30C45, 30C50.

Received: October, 2014.

Revised: November, 2014

Accepted: January, 2015. 
For $f \in \mathcal{A}$ of the form (1.1), we define the operator denoted $\mathcal{D}^{\lambda}, \lambda \in \mathbb{Z}=$ $\mathbb{N} \cup\{0\} \cup-\mathbb{N}=\{\cdots,-2,-1,0,1,2, \cdots\}$ by

$$
\mathcal{D}^{\lambda} f(z)=z+\sum_{k=1}^{\infty}(k+1)^{\lambda} a_{k+1} z^{k+1}, z \in \mathbb{U} .
$$

The operator $\mathcal{D}^{\lambda}$ was considered in [16] and for $\lambda \in \mathbb{N}_{0}=\mathbb{N} \cup\{0\}$ it is known as Sălăgean operator of order $\lambda$. In this case, it can be defined equivalently by $\mathcal{D}^{0} f(z)=f(z), \mathcal{D}^{1} f(z)=\mathcal{D} f(z)=z f^{\prime}(z), \mathcal{D}^{\lambda} f(z)=\mathcal{D}\left(\mathcal{D}^{\lambda-1} f(z)\right), \lambda \in \mathbb{N}$.

We note that $\mathcal{D}^{\lambda} \mathcal{D}^{-\lambda} f(z)=f(z)$, for all $\lambda \in \mathbb{Z}$.

Classes of analytic functions $f \in \mathcal{A}$ involving the quotient $\frac{\frac{z f^{\prime}(z)}{f(z)}}{\frac{f(z)}{z}}=\frac{z^{2} f^{\prime}(z)}{f^{2}(z)}$ have been studied in $[2,10,11,17,19]$. Also, the classes involving the quotient $\frac{\frac{z f^{\prime}(z)}{f(z)}}{\left(\frac{f(z)}{z}\right)^{\mu}}=f^{\prime}(z)\left(\frac{z}{f(z)}\right)^{1+\mu}$ have been studied for $\mu>-1$ in [13] (for $-1<\mu<$ 0 in [9] and for $0<\mu<1$ in [22]). Moreover, for $\mu \neq 0$, a class involving a certain linear operator under a subordination condition is investigated in [4]. Interestingly, a combination of both $f^{\prime}(z)\left(\frac{z}{f(z)}\right)^{1+\mu}$ and $\left(\frac{z}{f(z)}\right)^{\mu}$ for $0<\mu<1$ was studied in [23] (see also [18, Definition 1.1, p. 5]).

It may be observed that the operator $\mathcal{D}^{\lambda}$ preserves the class $\mathcal{A}$ and hence $\mathcal{D}^{\lambda} f(z)=0$ at $z=0$. Let $\lambda \in \mathbb{N}_{0}$ and let $f \in \mathcal{A}$ be such that $\mathcal{D}^{\lambda} f(z) \neq 0$ for $z \in \mathbb{U} \backslash\{0\}$. We define a function $q(z)$ by

$$
q(z)=\left(\frac{z}{\mathcal{D}^{\lambda} f(z)}\right)^{\mu-2} \quad(\mu \geq 0, \quad \mu \neq 2, \quad z \in \mathbb{U} \backslash\{0\}) \text { and } q(0)=1,
$$

where we assume that only principal values of $\left(\frac{z}{\mathcal{D}^{\lambda} f(z)}\right)^{\mu-2}$ are taken into consideration. Clearly, the function $q(z)$ is analytic in the open unit disk $\mathbb{U}$.

Recently, by considering the expression $q(z) \frac{\mathcal{D}^{\lambda+1} f(z)}{\mathcal{D}^{\lambda} f(z)}, \mu \geq 0$, Prajapat and Raina [14] investigated a class $\mathcal{B}(\lambda, \mu ; \alpha)$ of functions $f \in \mathcal{A}$ satisfying the condition that

$$
\left|q(z) \frac{\mathcal{D}^{\lambda+1} f(z)}{\mathcal{D}^{\lambda} f(z)}-1\right|<1-\alpha, \mu \geq 0,0 \leq \alpha<1, z \in \mathbb{U} .
$$

It may be noted that for $\lambda=0, \alpha=0, \mu=3$, the class $\mathcal{B}(0,3 ; 0)=\mathcal{U}$ was earlier studied by Ozaki and Nunukawa in [11] (see also Obradovic et al. [10] and Singh [19]), where it is proved that the functions $f \in \mathcal{U}$ are univalent.

For two analytic functions $p, q$ such that $p(0)=1=q(0)$, we say that $p$ is subordinate to $q$ in $\mathbb{U}$ and write $p(z) \prec q(z), z \in \mathbb{U}$, if there exists a Schwarz 
function $w$, analytic in $\mathbb{U}$ with $w(0)=0$, and $|w(z)|<1, z \in \mathbb{U}$ such that $p(z)=q(w(z)), z \in \mathbb{U}$. Furthermore, if the function $q$ is univalent in $\mathbb{U}$, then we have the following equivalence:

$$
p(z) \prec q(z) \Leftrightarrow p(0)=q(0) \text { and } p(\mathbb{U}) \subset q(\mathbb{U}) .
$$

Janowski [5] defined a class $\mathcal{P}(A, B)$ of analytic functions $p(z), z \in \mathbb{U}$, with $p(0)=1$, if $p(z) \prec \frac{1+A z}{1+B z},-1 \leq B<A \leq 1, z \in \mathbb{U}$. If $p \in \mathcal{P}(A, B)$, then it follows that

$$
\left|p(z)-\frac{1-A B}{1-B^{2}}\right|<\frac{A-B}{1-B^{2}} \text { for }-1<B<A \leq 1, z \in \mathbb{U}
$$

and for $B=-1$,

$$
\Re(p(z))>\frac{1-A}{2},-1<A \leq 1, z \in \mathbb{U} .
$$

The class $\mathcal{P}(1,-1)=\mathcal{P}$ is a Carathéodory class of functions which are analytic with positive real part in $\mathbb{U}$.

In this paper, we consider a new class $\mathcal{L}(\lambda, \mu ; \phi)$ of analytic functions (which evidently generalizes the class $\mathcal{B}(\lambda, \mu ; \alpha)$ ) comprising of functions $f \in$ $\mathcal{A}$ if and only if (for $\frac{z}{\mathcal{D}^{\lambda} f(z)} \neq 0$ in $\left.\mathbb{U}\right)$ :

$$
q(z) \frac{\mathcal{D}^{\lambda+1} f(z)}{\mathcal{D}^{\lambda} f(z)} \prec \phi(z)\left(\lambda \in \mathbb{N}_{0}, \mu \geq 0 ; z \in \mathbb{U}\right),
$$

where $q(z)$ is given by $(1.2), \mathcal{D}^{\lambda}$ is the Sălăgean operator and $\phi \in \mathcal{P}$ is a convex function in $\mathbb{U}$; see also the works in [20] and [21].

We note that $\mathcal{L}(0,2 ; \phi)=S^{*}[\phi]$ and $\mathcal{L}(1,2 ; \phi)=K[\phi]$ are the classes introduced by Ma and Minda [7] which include several well-known starlike and convex mappings as special cases.

For the bilinear transformation $\phi(z)=\frac{1+A z}{1+B z}(-1 \leq B<A \leq 1, z \in \mathbb{U})$, we denote $\mathcal{L}\left(\lambda, \mu ; \frac{1+A z}{1+B z}\right)$ by $\mathcal{T}(\lambda, \mu ; A, B)$.

We observe that the class $\mathcal{T}(\lambda, 2 ; A, B)=\mathcal{P}_{\lambda}^{\lambda+1}(A, B)$ was earlier considered by Kuroki and Owa [6, Remark 2, p. 4] for any integer $\lambda$, and for complex parameters $A$ and $B$, the class $\mathcal{T}(0,3 ; A, B)=\mathcal{T}(A, B)$ was studied by Shanmugam and Gangadharan [17]. The class $\mathcal{T}(0,2 ; A, B)=\mathcal{S}(A, B)$ is the class of Janowski starlike functions [5]. Further, the classes $\mathcal{T}(\lambda, \mu ; 1-\alpha, 0)=$ $\mathcal{B}(\lambda, \mu ; \alpha)$ and $\mathcal{T}(0,3 ; 1-\alpha, 0)=\mathcal{B}(\alpha)(0 \leq \alpha<1)$ were studied in [2] and various subordination properties and sufficient conditions were investigated in these classes of functions. 
For the purpose of this paper, we consider the functions $f \in \mathcal{A}$ of the form (1.1) such that the coefficients $b_{k}(k \in \mathbb{N})$ defined by

$$
q(z)=\left(\frac{z}{\mathcal{D}^{\lambda} f(z)}\right)^{\mu-2}=1+\sum_{k=1}^{\infty} b_{k} z^{k}, z \in \mathbb{U}
$$

to be non-negative.

Example 1. Let $\mu \geq 0, \mu \neq 2$ and let $\lambda \in \mathbb{N}_{0}$; if we consider $f \in \mathcal{A}$ of the form $f(z)=\mathcal{D}^{-\lambda}\left(z e^{z /(2-\mu)}\right)$, then $q(z)=e^{z}$ has the form (1.5).

Example 2. Let $0<\mu<2, \mu=\frac{p}{r}, p, r \in \mathbb{N}$ and let $\lambda=1$; if

$$
f(z)=\frac{1}{r+1}\left[(1+z)^{r+1}-1\right] \text {, then } q(z)=(1+z)^{2 r-p} .
$$

Example 3. Let $\mu>2$ and let $\lambda \in \mathbb{N}_{0}$; if we consider $f \in \mathcal{A}, f(z)=z-a_{n} z^{n}$, where $a_{n}>0$ and $n \geq 2$, then

$$
\begin{gathered}
q(z)=\left[1-n^{\lambda} a_{n} z^{n-1}\right]^{2-\mu}= \\
1+\sum_{k=1}^{\infty} \frac{(-\mu+2)(-\mu+1)(-\mu) \cdots(-\mu-(k-3))}{k !}\left(-n^{\lambda} a_{n} z^{n-1}\right)^{k}= \\
1+\sum_{k=1}^{\infty} \frac{(\mu-2)(\mu-1)(\mu) \cdots(\mu+k-3)}{k !} n^{k \lambda}\left(a_{n}\right)^{k} z^{k(n-1)} .
\end{gathered}
$$

Example 4. Let $\mu \geq 0, \mu \neq 2$ and let $\lambda \in \mathbb{N}_{0}$; if we consider $f \in \mathcal{A}$ of the form $f(z)=\mathcal{D}^{-\lambda}\left(z(1+z)^{1 /(2-\mu)}\right)$, then $q(z)=1+z$.

Example 5. Evidently, for $f$ of the form (1.1) with $a_{k+1} \geq 0$ and for $\mu=1$ and $\lambda \in \mathbb{N}_{0}$, the coefficients $b_{k}$ are given by $b_{k}=(k+1)^{\lambda} a_{k+1}, k \in \mathbb{N}$.

In this paper, we concentrate ourselves in investigating some basic characteristic properties such as the coefficient inequality, the radius result, subordination and inclusion properties for the functions $f \in \mathcal{T}(\lambda, \mu ; A, B)$. Sharp bounds for the initial coefficient, the Fekete-Szegö functional of functions $f(z)$ and integral representations belonging to this class are also determined.

\section{A Coefficient Inequality}

We begin to investigate the coefficient inequality of functions $f \in \mathcal{T}(\lambda, \mu ; A, B)$, which is contained in the following: 
Theorem 1. Let $-1 \leq B<A \leq 1, \mu \in[1,3] \backslash\{2\}$, let $f \in \mathcal{A}$ of the form (1.1) and let $b_{k}, k \in \mathbb{N}$ defined by (1.5) be non-negative. If

$$
\sum_{k=1}^{\infty} \frac{k-\mu+2}{|\mu-2|} b_{k} \leq \frac{A-B}{1+|B|}
$$

then $f \in \mathcal{T}(\lambda, \mu ; A, B)$. The condition (2.1) is necessary for $f \in \mathcal{T}(\lambda, \mu ; A, B)$ provided that $-1 \leq B \leq 0<A \leq 1, \mu \in(2,3]$.

Proof. Let

$$
p(z)=q(z) \frac{\mathcal{D}^{\lambda+1} f(z)}{\mathcal{D}^{\lambda} f(z)}, z \in \mathbb{U},
$$

where $q(z)$ is given by (1.2) then, we get

$$
p(z)=q(z)-\frac{z q^{\prime}(z)}{\mu-2} .
$$

Since $f \in \mathcal{T}(\lambda, \mu ; A, B)$, if and only if

$$
\left|\frac{p(z)-1}{A-B p(z)}\right|<1, z \in \mathbb{U}
$$

therefore, if we consider

$$
P=|p(z)-1|-|A-B p(z)|,
$$

then in view of (1.5) and (2.3), we get

$$
\begin{aligned}
P & =\left|-\sum_{k=1}^{\infty} \frac{k-\mu+2}{\mu-2} b_{k} z^{k}\right|-\left|A-B+\sum_{k=1}^{\infty} \frac{k-\mu+2}{\mu-2} B b_{k} z^{k}\right| \\
& <\sum_{k=1}^{\infty} \frac{k-\mu+2}{|\mu-2|} b_{k}-\left[A-B-\sum_{k=1}^{\infty} \frac{k-\mu+2}{|\mu-2|}|B| b_{k}\right] \\
& =\sum_{k=1}^{\infty} \frac{k-\mu+2}{|\mu-2|}(1+|B|) b_{k}-(A-B) \leq 0,
\end{aligned}
$$

on using (2.1). For the necessary part, we consider for $-1 \leq B \leq 0<A \leq 1$, $\mu \in(2,3]$ that $f \in \mathcal{T}(\lambda, \mu ; A, B)$, then from (2.4), in view of (1.5) and (2.3), we have

$$
\left|\frac{-\sum_{k=1}^{\infty} \frac{k-\mu+2}{\mu-2} b_{k} z^{k}}{A-B-\sum_{k=1}^{\infty} \frac{k-\mu+2}{\mu-2}|B| b_{k} z^{k}}\right|<1, z \in \mathbb{U} .
$$


Since $p(z)$ in $(2.3)$ is real for real $z$, letting $z \rightarrow 1^{-}$along real axis, we get from the condition that

$$
\Re(p(z))>\frac{1-A}{1-B}
$$

which ensures that the denominator under the mod sign in the inequality (2.5) remains positive and then we have

$$
\frac{\sum_{k=1}^{\infty} \frac{k-\mu+2}{\mu-2} b_{k}}{A-B-\sum_{k=1}^{\infty} \frac{k-\mu+2}{\mu-2}|B| b_{k}} \leq 1
$$

which proves (2.1). This completes the proof of Theorem 1 .

From Theorem 1, for the cases when $B=0$ and $B=-1(\mu \in(2,3])$, respectively, and applying the well-known assertions (1.3) and (1.4), we get the following results.

Corollary 1. Let $0<A \leq 1, \mu \in(2,3]$ and let $f \in \mathcal{A}$ of the form (1.1) and let $b_{k}, k \in \mathbb{N}$ defined by (1.5) be non-negative. Then

$$
\left|q(z) \frac{\mathcal{D}^{\lambda+1} f(z)}{\mathcal{D}^{\lambda} f(z)}-1\right|<A, z \in \mathbb{U},
$$

if and only if

$$
\sum_{k=1}^{\infty} \frac{k-\mu+2}{\mu-2} b_{k} \leq A .
$$

Corollary 2. Let $-1<A \leq 1, \mu \in(2,3]$ and let $f \in \mathcal{A}$ of the form (1.1) and let $b_{k}, k \in \mathbb{N}$ defined by (1.5) be non-negative. Then

$$
\Re\left(q(z) \frac{\mathcal{D}^{\lambda+1} f(z)}{\mathcal{D}^{\lambda} f(z)}\right)>\frac{1-A}{2}, z \in \mathbb{U},
$$

if and only if

$$
\sum_{k=1}^{\infty} \frac{k-\mu+2}{\mu-2} b_{k} \leq \frac{1+A}{2} .
$$

Remark 1. For $\lambda=0, \mu=3, A=1$, Corollary 1 corresponds to the known result of Ponnusamy and Sahoo [13, Theorem 7, p. 400]. 


\section{Radius Result}

Theorem 2. Let $-1 \leq B<A \leq 1, \mu \in[1,3] \backslash\{2\}$ and let $f \in \mathcal{A}$ of the form (1.1) and let $b_{k}, k \in \mathbb{N}$ defined by (1.5) be non-negative and satisfy the condition that

$$
\sum_{k=1}^{\infty} \frac{k-\mu+2}{|\mu-2|}\left(b_{k}\right)^{2} \leq 1
$$

Then

$$
\frac{1}{r} f(r z) \in \mathcal{T}(\lambda, \mu ; A, B)
$$

for $0<r \leq r_{0}$, where $r_{0}=r_{0}(\mu, A, B)$ is given by

$$
r_{0}=\frac{\eta \sqrt{2|\mu-2|}}{\left[3-\mu+2 \eta^{2}|\mu-2|+E\right]^{1 / 2}}
$$

where $E=\sqrt{\left\{3-\mu+2 \eta^{2}|\mu-2|\right\}^{2}+4 \eta^{2}|\mu-2|\left(\mu-2-\eta^{2}|\mu-2|\right)}$ and $\eta=$ $\frac{A-B}{1+|B|}$.

Proof. Let $f \in \mathcal{A}$ be of the form (1.1) with $\mu \in[1,3] \backslash\{2\}$. Then for $0<r \leq 1$, we have

$$
q(r z)=\left(\frac{z}{\frac{1}{r} \mathcal{D}^{\lambda} f(r z)}\right)^{\mu-2}=1+\sum_{k=1}^{\infty} b_{k} r^{k} z^{k}, b_{k} \geq 0, z \in \mathbb{U},
$$

where $q(z)$ is given by (1.2). Thus, by Theorem $1, \frac{1}{r} f(r z) \in \mathcal{T}(\lambda, \mu ; A, B)$ if

$$
R:=\sum_{k=1}^{\infty} \frac{k-\mu+2}{|\mu-2|} b_{k} r^{k} \leq \frac{A-B}{1+|B|} .
$$

By Cauchy-Schwarz inequality and the condition (3.1), we obtain that

$$
\begin{aligned}
R & \leq\left(\sum_{k=1}^{\infty} \frac{k-\mu+2}{|\mu-2|}\left(b_{k}\right)^{2}\right)^{1 / 2}\left(\sum_{k=1}^{\infty} \frac{k-\mu+2}{|\mu-2|} r^{2 k}\right)^{1 / 2} \\
& \leq \frac{1}{\sqrt{|\mu-2|}}\left(\sum_{k=1}^{\infty}(k-\mu+2) r^{2 k}\right)^{1 / 2} \\
& =\frac{1}{\sqrt{|\mu-2|}}\left(\frac{r^{4}}{\left(1-r^{2}\right)^{2}}+(3-\mu) \frac{r^{2}}{1-r^{2}}\right)^{1 / 2} \\
& =\frac{1}{\sqrt{|\mu-2|}} \frac{r}{1-r^{2}}\left\{3-\mu+(\mu-2) r^{2}\right\}^{1 / 2} \leq \frac{A-B}{1+|B|},
\end{aligned}
$$


provided that the inequality

$$
\frac{r}{1-r^{2}}\left\{3-\mu+(\mu-2) r^{2}\right\}^{1 / 2} \leq \eta \sqrt{|\mu-2|}
$$

holds, where $\frac{A-B}{1+|B|}=\eta$, or equivalently

$$
\frac{1}{r^{4}} \eta^{2}|\mu-2|-\frac{1}{r^{2}}\left\{3-\mu+2 \eta^{2}|\mu-2|\right\}-\left\{\mu-2-\eta^{2}|\mu-2|\right\} \geq 0
$$

holds, which provides the value of $r_{0}$ given by (3.2). This proves Theorem 2 .

Remark 2. By setting $\lambda=0, \mu=3-\alpha(0 \leq \alpha<1)$ and $B=0, A=\eta$, Theorem 2 coincides with the result of Ponnusamy and Sahoo [13, Theorem 5, p. 398] for univalent functions $f(z)$.

\section{Subordination Result}

Theorem 3. Let $-1 \leq B<A \leq 1, \mu \in[1,2)$ and let $f \in \mathcal{A}$ of the form (1.1) and let $b_{k}, k \in \mathbb{N}$ defined by (1.5) be non-negative. If $f \in \mathcal{T}(\lambda, \mu ; A, B)$, then

$$
\left(\frac{z}{\mathcal{D}^{\lambda} f(z)}\right)^{\mu-2} \prec \frac{1+A z}{1+B z}, z \in \mathbb{U}
$$

and hence,

$$
b_{k} \leq A-B, k \in \mathbb{N} .
$$

Proof. Let $q(z)$ be defined by (1.2), which is analytic in $\mathbb{U}$ with $q(0)=1$, then from $(2.3)$, we have

$$
q(z)+\frac{z q^{\prime}(z)}{2-\mu} \prec \frac{1+A z}{1+B z}, z \in \mathbb{U},
$$

which by the result of Hallenbeck and Ruscheweyh [3] proves (4.1). Further, on using a well-known result of Rogosinski [15] on subordination, and in view of (1.5), the subordination (4.1) gives the coefficient inequality (4.2).

\section{Inclusion Result}

Theorem 4. Let $\lambda \in \mathbb{N}_{0},-1 \leq B \leq 0<A \leq 1, \mu \in(2,3]$ and let $f \in \mathcal{A}$ of the form (1.1) and let $b_{k}, k \in \mathbb{N}$ defined by (1.5) be non-negative. If

$$
\frac{\mathcal{D}^{\lambda+1} f(z)}{\mathcal{D}^{\lambda} f(z)} \prec \frac{1+A z}{1+B z}, z \in \mathbb{U},
$$


then

$$
\sum_{k=1}^{\infty}\left\{\frac{k-\frac{A-B}{1+|B|}(\mu-2)}{\mu-2}\right\} b_{k} \leq \frac{A-B}{1+|B|} .
$$

Hence, $\mathcal{P}_{\lambda}^{\lambda+1}(A, B) \subset \mathcal{T}(\lambda, \mu ; A, B)$.

Proof. From (5.1), we have

$$
f \in \mathcal{P}_{\lambda}^{\lambda+1}(A, B) \Leftrightarrow \frac{\mathcal{D}^{\lambda+1} f(z)}{\mathcal{D}^{\lambda} f(z)} \prec \frac{1+A z}{1+B z} \Leftrightarrow\left|\frac{\frac{\mathcal{D}^{\lambda+1} f(z)}{\mathcal{D}^{\lambda} f(z)}-1}{A-B \frac{\mathcal{D}^{\lambda+1} f(z)}{\mathcal{D}^{\lambda} f(z)}}\right|<1, z \in \mathbb{U} .
$$

Let $q(z)$ be defined by (1.2), then on using (2.2) and (2.3), we get

$$
\frac{z q^{\prime}(z)}{q(z)}=(\mu-2)\left(1-\frac{\mathcal{D}^{\lambda+1} f(z)}{\mathcal{D}^{\lambda} f(z)}\right) .
$$

Hence, by (1.5), the condition (5.3) can equivalently be expressed as

$$
\left|\frac{-\sum_{k=1}^{\infty} k b_{k} z^{k}}{(A-B)(\mu-2)\left(1+\sum_{k=1}^{\infty} b_{k} z^{k}\right)+B \sum_{k=1}^{\infty} k b_{k} z^{k}}\right|<1, z \in \mathbb{U} .
$$

Since $\frac{\mathcal{D}^{\lambda+1} f(z)}{\mathcal{D}^{\lambda} f(z)}$ is real for real $z$, letting $z \rightarrow 1^{-}$along the real axis, we get from (5.1) that

$$
\Re\left(\frac{\mathcal{D}^{\lambda+1} f(z)}{\mathcal{D}^{\lambda} f(z)}\right)>\frac{1-A}{1-B} \Leftrightarrow \frac{\mathcal{D}^{\lambda+1} f(z)}{\mathcal{D}^{\lambda} f(z)}>\frac{1-A}{1-B}
$$

and hence, for being $B \leq 0$,

$$
A-B \frac{\mathcal{D}^{\lambda+1} f(z)}{\mathcal{D}^{\lambda} f(z)}>A+|B| \frac{1-A}{1-B}>0,
$$

which ensures that the denominator under the mod sign in the inequality (5.4) is positive. Thus, we have

$$
\frac{\sum_{k=1}^{\infty} k b_{k}}{(A-B)(\mu-2)-\sum_{k=1}^{\infty}\{|B| k-(A-B)(\mu-2)\} b_{k}} \leq 1,
$$


which yields the desired inequality (5.2). Further, since $\frac{A-B}{1+|B|} \leq 1$, if $f \in \mathcal{P}_{\lambda}^{\lambda+1}(A, B)$, we have by (5.2) that

$$
\sum_{k=1}^{\infty} \frac{k-\mu+2}{\mu-2} b_{k} \leq \sum_{k=1}^{\infty}\left\{\frac{k-\frac{A-B}{1+|B|}(\mu-2)}{\mu-2}\right\} b_{k} \leq \frac{A-B}{1+|B|},
$$

and consequently by Theorem 1 , we conclude that $f \in \mathcal{T}(\lambda, \mu ; A, B)$. This proves the inclusion result.

\section{Fekete-Szegö Problem}

Let $f(z)$ of the form (1.1) be in the class $\mathcal{T}(\lambda, \mu ; A, B)$, then for some Schwarz function $w(z)$, we get

$$
q(z) \frac{\mathcal{D}^{\lambda+1} f(z)}{\mathcal{D}^{\lambda} f(z)}=\frac{1+A w(z)}{1+B w(z)}, z \in \mathbb{U},
$$

where $q(z)$ is given by (1.2) and upon using the series:

$$
\mathcal{D}^{\lambda} f(z)=z+\sum_{k=1}^{\infty}(k+1)^{\lambda} a_{k+1} z^{k+1}, z \in \mathbb{U}
$$

and performing elementary calculations, we can write the series expansion

$$
\begin{aligned}
& q(z) \frac{\mathcal{D}^{\lambda+1} f(z)}{\mathcal{D}^{\lambda} f(z)} \\
= & \frac{\mathcal{D}^{\lambda+1} f(z)}{z}\left(\frac{z}{\mathcal{D}^{\lambda} f(z)}\right)^{\mu-1} \\
= & 1+(3-\mu) 2^{\lambda} a_{2} z+(4-\mu)\left\{3^{\lambda} a_{3}-(\mu-1) 2^{2 \lambda-1} a_{2}^{2}\right\} z^{2}+\ldots
\end{aligned}
$$

For the Schwarz function $w(z)$, let $\phi \in \mathcal{P}$ be defined by

$$
\phi(z)=\frac{1+w(z)}{1-w(z)}=1+c_{1} z+c_{2} z^{2}+\ldots
$$

Then

$$
\frac{1+A w(z)}{1+B w(z)}=1+\frac{A-B}{2} c_{1} z+\frac{A-B}{2}\left\{c_{2}-\frac{B+1}{2} c_{1}^{2}\right\} z^{2}+\ldots
$$

and from (6.2) and (6.4), we get

$$
\begin{aligned}
(3-\mu) 2^{\lambda} a_{2} & =\frac{A-B}{2} c_{1}, \\
(4-\mu)\left\{3^{\lambda} a_{3}-(\mu-1) 2^{2 \lambda-1} a_{2}^{2}\right\} & =\frac{A-B}{2}\left\{c_{2}-\frac{B+1}{2} c_{1}^{2}\right\} .
\end{aligned}
$$


In order to find in this section sharp upper bound for $\left|a_{2}\right|$ and for the FeketeSzegö functional $\left|a_{3}-\rho a_{2}^{2}\right|(\rho \in \mathbb{C})$, we use the following result from [12, p. 166] (see also [1, p. 41]).

Lemma 1. Let $\phi \in \mathcal{P}$ be of the form $\phi(z)=1+c_{1} z+c_{2} z^{2}+\ldots$, then

$$
\left|c_{2}-c_{1}^{2} / 2\right| \leq 2-\left|c_{1}\right|^{2} / 2
$$

and $\left|c_{k}\right| \leq 2$ for all $k \in \mathbb{N}$.

Theorem 5. Let $-1 \leq B<A \leq 1, \mu \in(2,3)$, and $f \in \mathcal{A}$ be of the form (1.1) belong to the class $\mathcal{T}(\lambda, \mu ; A, B)$, then

$$
\left|a_{2}\right| \leq \frac{A-B}{(3-\mu) 2^{\lambda}}
$$

and for all $\rho \in \mathbb{C}$ :

$\left|a_{3}-\rho a_{2}^{2}\right| \leq \frac{A-B}{(4-\mu) 3^{\lambda}} \max \left\{1,\left|\left(\frac{\mu-1}{3^{\lambda}} 2^{2 \lambda-1}-\rho\right) \frac{(A-B)(4-\mu) 3^{\lambda}}{(3-\mu)^{2} 2^{2 \lambda}}-B\right|\right\}$.

The result is sharp if $B=-1$ or if $B=0$.

Proof. Let the function $f(z)$ of the form (1.1) belong to the class $\mathcal{T}(\lambda, \mu ; A, B)$, then using the Carathéodory condition: $\left|c_{1}\right| \leq 2$ in (6.5), for the functions $\phi \in \mathcal{P}$ of the form (6.3), we get

$$
\left|a_{2}\right| \leq \frac{A-B}{(3-\mu) 2^{\lambda}},
$$

which by virtue of (6.5) and (6.6) gives

$$
\begin{aligned}
a_{3}-\rho a_{2}^{2}= & \left(\frac{(\mu-1) 2^{2 \lambda-1}}{3^{\lambda}}-\rho\right) \frac{(A-B)^{2}}{4(3-\mu)^{2} 2^{2 \lambda}} c_{1}^{2}+ \\
& +\frac{A-B}{2(4-\mu) 3^{\lambda}}\left\{c_{2}-\frac{B+1}{2} c_{1}^{2}\right\}=\frac{A-B}{2(4-\mu) 3^{\lambda}}\left(c_{2}-\frac{1}{2} c_{1}^{2}\right)+ \\
& +\left(\left(\frac{\mu-1}{3^{\lambda}} 2^{2 \lambda-1}-\rho\right) \frac{(A-B)^{2}}{(3-\mu)^{2} 2^{2 \lambda+2}}-\frac{(A-B) B}{4(4-\mu) 3^{\lambda}}\right) c_{1}^{2} .
\end{aligned}
$$

By Lemma 1, it follows that

$$
\left|a_{3}-\rho a_{2}^{2}\right| \leq F\left(\left|c_{1}\right|\right)=C+C D \frac{\left|c_{1}\right|^{2}}{4},
$$


where

$C=\frac{(A-B)}{(4-\mu) 3^{\lambda}}>0, D=|E|-1, E=\left(\frac{\mu-1}{3^{\lambda}} 2^{2 \lambda-1}-\rho\right) \frac{(A-B)(4-\mu) 3^{\lambda}}{(3-\mu)^{2} 2^{2 \lambda}}-B$.

As $\left|c_{1}\right| \leq 2$, we infer that

$$
\left|a_{3}-\rho a_{2}^{2}\right| \leq\left\{\begin{array}{cc}
F(0)=C, & |E| \leq 1 \\
F(2)=C|E| & |E| \geq 1
\end{array}\right.
$$

In the case when $B=-1$, the sharpness can be verified for the functions given by

$$
q(z) \frac{\mathcal{D}^{\lambda+1} f(z)}{\mathcal{D}^{\lambda} f(z)}=\frac{1+A z^{2}}{1-z^{2}}\left(\text { or } \frac{1+A z}{1-z}\right), z \in \mathbb{U}
$$

and, in case when $B=0$, the sharpness can be verified for functions given by

$$
q(z) \frac{\mathcal{D}^{\lambda+1} f(z)}{\mathcal{D}^{\lambda} f(z)}=1+A z^{2}(\text { or } 1+A z), z \in \mathbb{U}
$$

where $q(z)$ is given by (1.2). This completes the proof of Theorem 5 .

Remark 3. For $\lambda=0, \mu=2+\nu(0<\nu<1)$, Theorem 5 corresponds (for $A=1-2 \alpha(0 \leq \alpha<1), B=-1)$ to Theorem 1 , and (for $A=k(0<k \leq 1)$, $B=0)$ to Theorem 2 of Tuneski and Darus [22, pp. 64-65].

\section{$7 \quad$ Integral Representations}

Theorem 6. Let $-1 \leq B<A \leq 1,2<\mu \leq 3$ and $f \in \mathcal{A}$ be of the form (1.1). If $f \in \mathcal{T}(\lambda, \mu ; A, B)$, then for some Schwarz functions $w_{1}(z)$ and $w_{2}(z)$, $w_{1}(0)=0=w_{1}^{\prime}(0)-1($ in case $2<\mu<3)$ :

$$
\left(\frac{z}{\mathcal{D}^{\lambda} f(z)}\right)^{\mu-2}=1-(\mu-2)(A-B) z^{\mu-2} \int_{0}^{z} \frac{w_{1}(t)}{t^{\mu-1}\left(1+B w_{1}(t)\right)} d t, z \in \mathbb{U}
$$

and $w_{2}(0)=0=w_{2}^{\prime}(0)($ in case $\mu=3)$ :

$$
\frac{z}{\mathcal{D}^{\lambda} f(z)}=1-2^{\lambda} a_{2} z-(A-B) z \int_{0}^{z} \frac{w_{2}(t)}{t^{2}\left(1+B w_{2}(t)\right)} d t, z \in \mathbb{U}
$$


Proof. Let $f \in \mathcal{A}$ be of the form (1.1), then from (6.2), we have

$q(z) \frac{\mathcal{D}^{\lambda+1} f(z)}{\mathcal{D}^{\lambda} f(z)}=1+(3-\mu) 2^{\lambda} a_{2} z+(4-\mu)\left\{3^{\lambda} a_{3}-(\mu-1) 2^{2 \lambda-1} a_{2}^{2}\right\} z^{2}+\ldots$,

where $q(z)$ is given by (1.2). Hence, if $f \in \mathcal{T}(\lambda, \mu ; A, B)$, the Schwarz function $w(z)$ in $(6.1)$ is given by

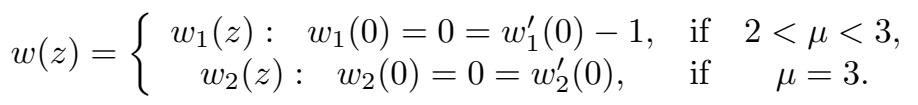

It is easy to verify that

$$
\frac{d}{d z}\left(\frac{1}{\left(\mathcal{D}^{\lambda} f(z)\right)^{\mu-2}}-\frac{1}{z^{\mu-2}}\right)=-\frac{\mu-2}{z^{\mu-1}}\left(q(z) \frac{\mathcal{D}^{\lambda+1} f(z)}{\mathcal{D}^{\lambda} f(z)}-1\right),
$$

where $q(z)$ is given by (1.2) and therefore by (6.1), we get

$$
\frac{d}{d z}\left(\frac{1}{\left(\mathcal{D}^{\lambda} f(z)\right)^{\mu-2}}-\frac{1}{z^{\mu-2}}\right)=-\frac{(\mu-2)(A-B) w(z)}{z^{\mu-1}(1+B w(z))}, z \in \mathbb{U} .
$$

From (1.5), we also have

$$
\frac{1}{\left(\mathcal{D}^{\lambda} f(z)\right)^{\mu-2}}-\frac{1}{z^{\mu-2}}=\frac{1}{z^{\mu-2}}[q(z)-1]=\sum_{k=1}^{\infty} b_{k} z^{k-\mu+2},
$$

which yields that

$$
\left(\frac{1}{\left(\mathcal{D}^{\lambda} f(z)\right)^{\mu-2}}-\frac{1}{z^{\mu-2}}\right)_{z=0}=\left\{\begin{array}{cc}
b_{1}, & \mu=3 \\
0, & 2<\mu<3
\end{array} .\right.
$$

By using (1.5) and (6.2), and equating the coefficient of $z$ on both the sides of (2.3), we find that $b_{1}=-(\mu-2) 2^{\lambda} a_{2}$. Now, upon integrating (7.3), we obtain the desired representations given by (7.1) and (7.2).

Remark 4. For $\mu=3$ and $\lambda=0$, the above representation (7.2) corresponds to the representation due to Shanmugam and Gangadharan in [17, Theorem 2.1, pp. 2-3] and corresponds to Theorem 1 of Obradovic et al. [10] if $A=1$ and $B=0$. 
Corollary 3. Let $-1 \leq B \leq 0<A \leq 1$ and $f \in \mathcal{A}$ be of the form (1.1). If $f \in \mathcal{T}(\lambda, \mu ; A, B)$, then for $\mu \in(2,3)$ :

$$
\begin{aligned}
& \left|\left(\frac{z}{\mathcal{D}^{\lambda} f(z)}\right)^{\mu-2}-1\right| \\
\leq & \left\{\begin{array}{c}
\frac{(\mu-2) A}{3-\mu}|z|, B=0, z \in \mathbb{U}, \\
(\mu-2)(A-B) \frac{|z|}{3-\mu}{ }_{2} F_{1}(1,3-\mu, 4-\mu ;|B||z|),-1 \leq B<0, z \in \mathbb{U},
\end{array}\right.
\end{aligned}
$$

and for $\mu=3$ :

$$
\left|\frac{z}{\mathcal{D}^{\lambda} f(z)}-1\right| \leq\left\{\begin{array}{c}
2^{\lambda} a_{2}|z|+A|z|^{2}, B=0, z \in \mathbb{U} \\
2^{\lambda} a_{2}|z|+\frac{(A-B)|z|}{2 \sqrt{|B|}} \log \left(\frac{1+|z| \sqrt{|B|}}{1-|z| \sqrt{|B|}}\right),-1 \leq B<0, z \in \mathbb{U} .
\end{array}\right.
$$

Proof. From (7.1) when $\mu \in(2,3)$, and on substituting $t=z u$ and noting that

$\left|w_{1}(z u)\right| \leq|z| u$, we get

$$
\left|\left(\frac{z}{\mathcal{D}^{\lambda} f(z)}\right)^{\mu-2}-1\right| \leq(\mu-2)(A-B) \int_{0}^{1} \frac{|z|}{u^{\mu-2}(1-|B||z| u)} d u, z \in \mathbb{U} .
$$

Now if $B=0$, the above integral gives simply

$$
\int_{0}^{1} \frac{|z|}{u^{\mu-2}(1-|B||z| u)} d u=\frac{|z|}{3-\mu}, z \in \mathbb{U}
$$

and if $-1 \leq B<0$, making use of the known integral representation of the Gaussian hypergeometric function mentioned, for instance, see [8, p. 7], we get

$$
\int_{0}^{1} \frac{|z|}{u^{\mu-2}(1-|B||z| u)} d u=\frac{|z|}{3-\mu}{ }_{2} F_{1}(1,3-\mu, 4-\mu ;|B||z|)
$$

and hence, we have the inequality (7.4). Also, from (7.2), by substituting $t=z u$ and noting that $\left|w_{2}(z u)\right| \leq|z|^{2} u^{2}$, we get

$$
\left|\frac{z}{\mathcal{D}^{\lambda} f(z)}-1\right| \leq 2^{\lambda} a_{2}|z|+(A-B) \int_{0}^{1} \frac{|z|^{2}}{1-|B||z|^{2} u^{2}} d u, z \in \mathbb{U} .
$$


Using now the following integral (for $-1 \leq B \leq 0$ ):

$$
\int_{0}^{1} \frac{|z|^{2}}{1-|B||z|^{2} u^{2}} d u=\left\{\begin{array}{cc}
|z|^{2} & B=0, z \in \mathbb{U}, \\
\frac{|z|}{2 \sqrt{|B|}} \log \left(\frac{1+|z| \sqrt{|B|}}{1-|z| \sqrt{|B|}}\right), & -1 \leq B<0, z \in \mathbb{U},
\end{array}\right.
$$

we are lead to the second inequality (7.5) of Corollary 3.

Acknowledgment. The authors thank the reviewer for his/her valuable suggestions.

\section{References}

[1] P. L. Duren, Univalent Functions, Springer-Verlag, 1983.

[2] B. A. Frasin and M. Darus, On certain analytic univalent functions, Internat. J. Math. Math. Sci., 25 (5), (2001), 305-310.

[3] D. I. Hallenbeck, St. Ruscheweyh, Subordination by convex functions, Proc. Amer. Math. Soc., 52 (1975), 191-195.

[4] R. W. Ibrahim, M. Darus and N. Tuneski, On subordination for classes of non-Bazilevic type, Annales Univ. Mariae Curie-Sklodowska, 2 (2010), 49-60.

[5] W. Janowski, Some extremal problems for certain families of analytic functions I, Ann. Polon. Math., 28 (1973), 298-326.

[6] K. Kuroki and S. Owa, Some subordination criteria concerning the Sălăgean operator, J. Inequal. Pure and Appl. Math., 10 (2) (2009), Art. $36,11 \mathrm{pp}$.

[7] W. Ma and D. Minda, A unified treatment of some special classes of univalent functions, in: Proceedings of the Conference on Complex Analysis, Z. Li, F. Ren, L. Yang, and S. Zhang (Eds.), International Press (1994), $157-169$.

[8] S. S. Miller and P. T. Mocanu, Differential Subordinations: Theory and Applications, Pure and Applied Mathematics No. 225, Marcel Dekker, New York, 2000.

[9] M. Obradovic, A class of univalent functions, Hokkaido Math. J., 27 (2) (1998), 329-335. 
[10] M. Obradovic, N. N. Pascu and Radomir, A class of univalent functions, Math. Japon., 44 (3) (1996), 565-568.

[11] S. Ozaki and M. Nunokawa, The Schwarzian derivative and univalent functions, Proc. Amer. Math. Soc., 33 (2) (1972), 392-394.

[12] C. Pommerenke, Univalent Functions, in: Studia Mathematica Mathematische Lehrbucher, Vandenhoeck and Rupreht,1975.

[13] S. Ponnusamy and S. K. Sahoo, Study of some subclasses of univalent functions and their radius properties, Kodai Math. J., 29 (2006), 391405.

[14] J. K. Prajapat and R. K. Raina, Certain subclasses of analytic functions involving Sălăgean operator, Italian J. Pure Appld. Math., 27 (2010), 91-98.

[15] W. Rogosinski, On the coefficients of subordinate functions, Proc. London Math. Soc. (Ser.2), 48 (1943), 48-82.

[16] G. S. Sălăgean, Subclasses of univalent functions, in: Complex Analysis: Fifth Romanian-Finnish Seminar, Part 1 (Bucharest, 1981), in: Lecture Notes in Mathematics, vol. 1013, Springer-Verlag, Berlin, Heidelberg, New York, 1983, pp. 362-372.

[17] T. N. Shanmugam and A. Gangadharan, On a class of univalent funtions, J. Indian Acad. Math., 27 (1) (2005), 201-211.

[18] T. N. Shanmugam, M. P. Jeyaraman and S. Sivasubramanian, FeketeSzegö functional for some subclass of non-Bazilevic functions, J. Inequal. Pure Appld. Math., 7 (3), (2006), Art. 117, 16 pp.

[19] V. Singh, On a class of univalent functions, Internat. J. Math. Math. Sci., 23 (12), (2000), 855-857.

[20] H. M. Srivastava and Sevtap Sumer Eker, Some applications of a subordination theorem for a class of analytic functions, Appl. Math. Lett., 21 (2008), 394-399.

[21] H. M. Srivastava and Shigeyoshi Owa (Editors), Current Topics in Analytic Function Theory (xiv + 456 pp.). World Scientific Publishing Company, Singapore, New Jersey, London, and Hong Kong, 1992.

[22] N. Tuneski and M. Darus, Fekete-Szegö functional for non-Bazilevic functions, Acta Math. Acad. Paedagog. Nyhazi. (N.S.), 18 (2002), 63-65. 
[23] Z. Wang, C. Gao and M. Liao, On certain generalized class of nonBazilevic functions, Acta Math. Acad. Paedagog. Nyhazi. (N.S.), 21 (2005), 147-154.

R. K. RAINA,

M. P. University of Agriculture and Technology,

Udaipur 313001, Rajasthan, India,

Current address: 10/11 Ganpati Vihar, Opposite Sector 5, Udaipur 313002,

Rajasthan, India.

Email: rkraina_7@hotmail.com

Poonam SHARMA,

Department of Mathematics \&, Astronomy

University of Lucknow,

Lucknow 226007, India,.

Email: sharma_poonam@lkouniv.ac.in

Grigore Stefan. SĂLĂGEAN,

Department of Mathematics,

Faculty of Mathematics and Computer Science,

Babes-Bolyai University,

Str. M. Kogalniceanu Nr. 1, 400084 Cluj-Napoca, Romania.

Email: salagean@math.ubbcluj.ro 